\title{
Successful Healthcare Provider Strategies to Overcome Psychological Insulin Resistance in Japanese Patients with Type 2 Diabetes
}

\author{
Kentaro Okazaki - Tomotaka Shingaki (D) - Zhihong Cai • \\ Magaly Perez-Nieves · Lawrence Fisher
}

Received: March 27, 2019 / Published online: July 8, 2019

(C) The Author(s) 2019

\begin{abstract}
Introduction: EMOTION was a multinational, noninterventional study surveying current insulin-using adults with type 2 diabetes mellitus (T2D) who were initially reluctant to begin insulin treatment. In this Japanese population subanalysis of EMOTION, we identify the frequency and level of helpfulness of healthcare provider (HCP) actions, and we analyze life events ('actions/events') that assist T2D patients
\end{abstract}

Enhanced Digital Features To view enhanced digital features for this article go to https://doi.org/10.6084/ m9.figshare. 8332433 .

Electronic Supplementary Material The online version of this article (https://doi.org/10.1007/s13300019-0664-9) contains supplementary material, which is available to authorized users.

K. Okazaki

Nagoya University Graduate School of Medicine, 65 Tsurumai-cho, Showa-ku, Nagoya, Aichi 466-8550, Japan

T. Shingaki $(\bowtie) \cdot$ Z. Cai

Eli Lilly Japan K.K., 5-1-28 Isogami-dori, Chuo-ku,

Kobe, Hyogo 651-0086, Japan

e-mail: shingaki_tomotaka@lilly.com

M. Perez-Nieves

Eli Lilly and Company, 1555 S Harding Street, Indianapolis, IN 46221, USA

L. Fisher

Behavioral Diabetes Institute, 5405 Oberlin Drive

Suite 100, San Diego, CA 92121, USA with psychological insulin resistance in the decision to initiate insulin.

Methods: Participants were selected from Survey Sampling International and their local partners' market research panels in Japan. Quantitative surveys were administered between December 2016 and August 2017 to patients who met the study criteria. Participants were asked whether 45 actions/events occurred, and to rate the level of helpfulness of the actions/events in contributing to their decision to initiate insulin.

Results: Among the 594 eligible participating adults in the EMOTION study, 99 were from Japan. Despite initial reluctance to begin insulin treatment, $80.8 \%$ of the Japanese participants immediately commenced insulin. Practical demonstrations by HCPs on how to use insulin were rated by participants as most helpful. Examples of such practical demonstrations, reported as helping moderately or a lot, were 'HCP walked patient through the process of exactly how to take insulin' (82.8\%), 'HCP showed an insulin pen' (79.7\%), and 'HCP helped patient to see how simple it was to inject insulin' (79.1\%).

Conclusion: This study identifies actions that HCPs can use to assist Japanese patients in deciding whether to initiate insulin. These findings may aid the development of clinical interventions addressing reluctance to begin insulin treatment among Japanese patients with T2D. 
Funding: Eli Lilly and Company and Boehringer Ingelheim.

Plain Language Summary: Plain language summary available for this article.

\section{PLAIN LANGUAGE SUMMARY}

The increasing prevalence of type 2 diabetes mellitus (T2D) is a major health concern globally. In Japan, the prevalence of diabetes is predicted to increase over the next decades. Insulin is a hormone which keeps blood glucose levels within the normal range. People with diabetes often require insulin therapy as they do not make sufficient insulin, or the insulin that is produced does not work optimally. Many patients delay insulin therapy due to a variety of factors. Reluctance to begin insulin treatment is known as 'psychological insulin resistance' (PIR). Several studies have described recommendations to address PIR, but there is little research regarding effective strategies that help reluctant patients begin insulin. Reports describing PIR among Japanese patients are limited. EMOTION was a multinational study surveying insulin-using adults with T2D and initial PIR. We report a Japanese population subanalysis of EMOTION, identifying factors which help reluctant Japanese patients begin insulin therapy. Our results indicate that healthcare provider (HCP) actions are associated with a patient's decision to use insulin. Practical demonstrations by HCPs on how to use insulin were reported as most helpful. Reassurance concerning pain of injection, explanations regarding blood glucose numbers and future health, encouragement to make contact in case of any problems, and occurrence of a positive life event were also rated by Japanese participants as being helpful in deciding to commence insulin. This study provides important information on actions that can be used by HCPs to treat Japanese patients with T2D who are reluctant to begin insulin treatment.

Keywords: Insulin; Japan; Psychological insulin resistance; Type 2 diabetes mellitus

\section{INTRODUCTION}

The global incidence of diabetes has been steadily increasing over the past few decades [1]. In Japan, the increasing prevalence of diabetes, particularly type 2 diabetes mellitus (T2D), is a major health concern [2]. Additionally, the prevalence of diabetes in Japan is predicted to further increase over the next decades, mainly due to an aging population [2].

Poorly controlled diabetes may lead to a number of serious health complications [1]. Globally, treatment guidelines for T2D (World Health Organization, American Diabetes Association, and European Association for the Study of Diabetes) recommend a patient-centered and stepwise approach to managing glycemic control $[1,3]$. A focus on lifestyle management and diabetes self-management education and support are recommended, alongside metformin, oral antidiabetic and/or injectable medications, and/or basal insulin, as well as consultation with a healthcare provider (HCP) [3]. Similarly, guidelines for Japanese patients with diabetes recommend patient education regarding diet and exercise, followed by basal insulin therapy for those with inadequate glycemic control despite therapy with oral glucose-lowering medications [4]. This treatment strategy, however, should vary depending on the disease condition, age, metabolic abnormality, and status of diabetes complications of each patient [4].

While treatment with insulin is associated with higher rates of achieving adequate glycemic control [1,3], initiation of insulin therapy is often delayed due to a variety of physicianand patient-related factors [5]. Reluctance to initiate insulin treatment, known as 'psychological insulin resistance' (PIR), is common among adults with diabetes, with a recent study reporting $29.9 \%$ of insulin-naive adults with T2D declined insulin treatment after it was recommended by their HCP [6]. Recent studies revealed a high reluctance in the use of injectable therapies among injection-naïve Japanese patients with T2D, with less than $2 \%$ of study participants initially willing to take injectable medicines $[7,8]$. 
Numerous studies have examined psychological barriers preventing the initiation of insulin therapy in adults [5, 9-11]. PIR encompasses patient-related concerns, including injection anxiety, misconceptions about insulin, concerns about hypoglycemia, and societal attitudes [5, 10, 12, 13]. Several studies have documented recommendations to address PIR [14-17]; however, there is little research regarding factors or effective strategies that help reluctant patients begin insulin treatment. In addition, although there are reports describing PIR among Japanese diabetic patients [18-20], the evidence is limited.

To further understand the factors associated with reluctant patients initiating insulin, a multinational, noninterventional study (EMOTION) surveyed current insulin-using adults with T2D who were initially reluctant to begin insulin treatment. The EMOTION (AccEpting Insulin TreatMent for Reluctant PeOple with Type 2 DIabetes Mellitus-a GlObal Study to IdeNtify Effective Strategies) study was conducted in 3 phases: qualitative interviews (phase 1) with participants and HCPs in 6 countries (Brazil, Canada, Germany, Spain, the United Kingdom, and the United States), quantitative surveys (phase 2), and follow-up interviews (phase 3) with participants in the same 6 countries plus Japan. Phase 2 of EMOTION identified a number of HCP actions and life events (actions/events) that were rated as being the most helpful amongst the total population [21]. These included efforts to address injection concerns by demonstrating the insulin injection process, explaining the benefits of insulin, and adopting a collaborative communication style. The perceived helpfulness of these actions were, in turn, linked with earlier insulin initiation and greater insulin persistence over time [21].

We report a Japanese population subanalysis of EMOTION (phase 2). We aim to identify HCP actions/events that may help reluctant insulin users with T2D decide to initiate insulin therapy.

\section{METHODS}

The data used in this study were derived from the EMOTION study. EMOTION was conducted in 7 countries: Brazil, Canada, Japan, Germany, Spain, the United Kingdom, and the United States. The study protocol was approved by the Western Institutional Review Board (IRB), Puyallup, Washington, USA, as well as the Nagoya University IRB, Nagoya, Japan. All procedures performed in this study involving human participants were in accordance with the ethical standards of the Western IRB, Puyallup, Washington, USA, and the Nagoya University IRB, Nagoya, Japan, and with the 1964 Declaration of Helsinki and its later amendments or comparable ethical standards. Informed consent was obtained from all individual participants included in the study. Participants were compensated for their time. The study design has been previously described [21]. Briefly, qualitative interviews with a total of 29 insulin-using adults with T2D and 29 HCPs across 6 countries (Brazil, Canada, Germany, Spain, the United Kingdom, and the United States) were conducted to inform survey content and design. Phase 2 involved a 30-min quantitative survey, derived from the qualitative interviews, with participants with T2D from these same nations plus Japan. This report focuses on the findings of the quantitative survey in Japanese participants with T2D.

Participants were selected from the market research panels of Survey Sampling International (SSI) and their local partners in Japan. Surveys were translated for Japanese participants by professional linguists from TransPerfect with knowledge of the language, the subject area, and the target audience. Survey participants were compensated for their time.

Eligible participants were adults ( $\geq 21$ years old) with T2D who reported that they were initially not willing to start insulin treatment (i.e., chose "not willing" among the options "not willing," "slightly willing," "moderately willing," or "very willing"). Participants had been diagnosed with T2D at least 1 year before starting basal insulin (self-reported), started basal insulin between 30 days and 3 years before the survey, and had used any insulin regularly for the 30 days before the survey. Subjects were ineligible if they had been diagnosed with any other type of diabetes, had experience with insulin therapy before the initiation of basal 
insulin therapy, or if they had initiated insulin using a premix product or basal bolus therapy.

Quantitative surveys were administered to eligible participants between December 2016 and August 2017. Participants were given the survey via an online platform. Participants were asked to survey 45 actions and statements that assisted in insulin initiation and to denote whether each action occurred. Actions/events that occurred were then rated for helpfulness on a 4-point Likert scale: 1 = it didn't help at all, $2=$ it helped a little, $3=$ it helped moderately, $4=$ it helped a lot. Other questions in the following key areas were included: participant demographics; disease history; time from initial HCP consult to initiation of insulin; reasons for insulin reluctance; transformative point/person involved in initiation of insulin; role of HCP in initiating insulin; experiences post-insulin initiation satisfaction with treatment; and attitude regarding insulin use. Completed surveys were screened to identify and omit those that were problematic (e.g., surveys which were completed in $<10 \mathrm{~min}$ or had the same responses to all survey items pertaining to HCP messages and actions).

Findings were presented using descriptive summary statistics, which included the number and percentage of participants with specific responses to categorical questions, the means and standard deviations for continuous variables (including age and years since first diabetes diagnosis), and actions/events with Likertscale responses. The mean level of helpfulness of each item was calculated and rated from the most to the least helpful. Outcomes related to experiences post-insulin initiation were also summarized, including adherence to insulin therapy, experiences related to weight, energy, mood, and hypoglycemia, treatment satisfaction with insulin therapy, and attitude about insulin following insulin initiation.

\section{RESULTS}

\section{Participant Characteristics}

Of the 594 adults who were eligible to participate in phase 2 of the EMOTION study, 99 were from Japan. The mean age of the Japanese participants was 57.7 years [standard deviation (SD) 9.6]. Most participants were male (79.8\%), and the mean time from initial T2D diagnosis to first insulin use was 12.2 years (SD 7.7). The mean body mass index of Japanese participants was $25.4 \mathrm{~kg} / \mathrm{m}^{2}$ (SD 3.7). The mean hemoglobin A1c (HbA1c) at the time of insulin initiation was $9.4 \%$ (SD 2.0), while the most recent HbA1c was $7.8 \%$ (SD 1.2). Baseline participant demographics are presented in Table 1.

\section{Insulin Initiation}

Participants' thoughts and feelings prior to initiating insulin are outlined in Table 2. Participants were aware that taking insulin would help to maintain good control of blood glucose (76.8\%), and that taking insulin would help to improve their health (75.8\%). The most common negative thought amongst Japanese participants was that taking insulin meant that their diabetes had become much worse (72.7\%). Another common negative thought amongst Japanese participants was that the associated use of insulin meant that they had failed to manage their diabetes (62.6\%). Participants also reported being afraid of injecting themselves with a needle $(48.5 \%)$, and that the insulin recommendation meant that they would be required to take insulin forever (48.5\%).

Participants' reactions following their HCPs' recommendation to begin insulin, time to insulin initiation, and any insulin discontinuations (for $\geq 7$ days) are presented in Table 3 . Participants reported being moderately $(28.3 \%)$ or very surprised $(12.1 \%)$ by the recommendation to initiate insulin, with $29.3 \%$ and $13.1 \%$, respectively, being moderately or very upset. When insulin was recommended for the first time, $40.4 \%$ of participants indicated that they were slightly willing to initiate insulin therapy, while $59.6 \%$ were not willing at all. Despite this unwillingness, $80.8 \%$ of the participants immediately commenced regular insulin therapy upon first recommendation. The number of Japanese participants who reported one or more periods of discontinuing insulin for $\geq 7$ days was $8.1 \%$. 
Table 1 Participant characteristics

\begin{tabular}{|c|c|}
\hline Parameter & $(N=99)$ \\
\hline Age, mean $(\mathrm{SD})$ & $57.7(9.6)$ \\
\hline Gender, male, $n(\%)$ & $79(79.8)$ \\
\hline $\begin{array}{l}\text { Years from T2D diagnosis to } \\
\text { insulin use, mean (SD) }\end{array}$ & $12.2(7.7)$ \\
\hline $\operatorname{BMI}\left(\mathrm{kg} / \mathrm{m}^{2}\right)^{\mathrm{a}}$, mean $(\mathrm{SD})$ & $25.4(3.7)$ \\
\hline $\begin{array}{l}\text { Prior use of injectable diabetes } \\
\text { medications }, n(\%)\end{array}$ & $20(20.2)$ \\
\hline \multicolumn{2}{|c|}{ HbAlc value prior to insulin initiation ${ }^{c}$} \\
\hline $\mathrm{HbAlc}, \%$, mean $(\mathrm{SD})$ & $9.4(2.0)$ \\
\hline $\mathrm{HbAlc}, \mathrm{mmol} / \mathrm{mol}$, mean $(\mathrm{SD})$ & $78.8(22.1)$ \\
\hline \multicolumn{2}{|l|}{ Most recent $\mathrm{HbAlc}$ test $^{\mathrm{c}}$} \\
\hline HbAlc, \%, mean (SD) & $7.8(1.2)$ \\
\hline $\mathrm{HbAlc}, \mathrm{mmol} / \mathrm{mol}$, mean (SD) & $61.7(12.7)$ \\
\hline \multicolumn{2}{|l|}{ Year of insulin initiation (\%) } \\
\hline 2015 & 48.5 \\
\hline 2016 & 34.3 \\
\hline 2017 & 14.1 \\
\hline
\end{tabular}

$B M I$ body mass index, HbAlc hemoglobin A1c, GLP-1 glucagon-like peptide $1, N / n$ number of participants, $T 2 D$ type 2 diabetes mellitus, $S D$ standard deviation

${ }^{a}$ BMI categories were defined using the World Health Organization's Internal Classification

b Mainly indicates treatment with GLP-1 receptor agonist, not any insulin therapy

${ }^{c}$ Mean HbAlc was calculated among patients who had a test in the past year and knew the test result. HbAlc $\mathrm{mmol} / \mathrm{mol}$ was converted to $\%$ and vice versa according to the HbAlc conversion tables (Sept. 2011) of the New Zealand Society for the Study of Diabetes

\section{HCP Actions and Life Events Experienced by Japanese Participants}

The most frequently occurring HCP actions experienced by Japanese patients with T2D are displayed in Fig. 1. Overall, the most common HCP action was the advice that blood glucose levels would improve upon commencing insulin (96.0\%). The actions ranked second to fifth in occurrence were 'HCP helped the patient to see how simple it is to inject insulin' (91.9\%), 'HCP walked the patient through the process of how to use insulin' (87.9\%), 'HCP gave patient leaflets or other reading material about insulin' (85.9\%), and 'HCP helped patient to see that an insulin injection wasn't as painful as patient thought it might be' (83.8\%).

The HCP actions/events least commonly experienced by Japanese participants with T2D are displayed in Table $\mathrm{S} 1$ of the Electronic supplementary material (ESM), and include 'HCP helped patient to meet other people taking insulin' (8.1\%), 'A close friend or family member urged patient to go along with HCP recommendation to start insulin' (12.1\%), and 'HCP told patient that he/she could not continue to treat patient if patient refused to start insulin' (14.1\%).

\section{Most and Least Helpful HCP Actions and Life Events for Patients with T2D Initiating Insulin Treatment}

Actions/events that were most helpful in assisting participants to initiate insulin treatment are displayed in Fig. 2. The most helpful actions/events, reported as helping moderately or a lot, were 'HCP walked patient through the process of exactly how to take insulin' $(82.8 \%$, mean level of helpfulness 3.28), 'HCP showed an insulin pen' $(79.7 \%$, mean level of helpfulness 3.20), 'HCP helped patient to see how simple it was to inject insulin' $(79.1 \%$, mean level of helpfulness 3.19), 'HCP had patient try an injection himself/herself while patient was there in the office' $(75.0 \%$, mean level of helpfulness 3.14), and 'HCP gave an injection while patient was in the office' $(72.0 \%$, mean level of helpfulness 3.12).

Actions/events that were least helpful in assisting participants initiate insulin treatment are displayed in Table S1 of the ESM. The least helpful actions/events according to Japanese participants were 'HCP helped patient to meet other people who had already been taking insulin' (25.0\%, mean level of helpfulness 2.13), 
Table 2 Thoughts and feelings experienced by Japanese patients with T2D before initiating insulin treatment

Thoughts and feelings about insulin right before starting insulin regularly for the first time Frequency $^{\mathbf{a}}$ $n(\%)$

Taking insulin would help to maintain good control of blood glucose (sugar levels) $76(76.8)$

Taking insulin would help to improve my health

Taking insulin would mean my diabetes had become much worse

Taking insulin would help to prevent complications of diabetes

Taking insulin would mean that I had failed, that I hadn't done a good enough job taking care of my diabetes

I was afraid of injecting myself with a needle

Taking insulin would mean that I would need to take insulin forever

Taking insulin would mean that I had failed to manage my diabetes with diet and tablets

Taking insulin would mean that my diabetes would become a much more serious condition

Taking insulin would make me more dependent on my doctor

Taking insulin would mean other people would see me as a sick/sicker person

Injecting insulin would be painful

Taking insulin would increase the risk of low blood glucose levels (hypoglycemia)

It would be difficult to inject the right amount of insulin correctly at the right time every day

Managing insulin injections would take a lot of time and energy

Taking insulin would make life less flexible

Taking insulin would help to improve my energy level

Injecting insulin would be embarrassing

Being on insulin would cause family and friends to be more concerned about me

Insulin would cause weight gain

Taking insulin would mean I would have to give up activities I enjoy

Taking insulin might cause serious health problems such as blindness, kidney problems, or amputations

Taking insulin would make it more difficult to fulfill my responsibilities (at home and work)

Taking insulin would mean my health would deteriorate

Taking insulin would cause me to lose my job

a Frequency was calculated using responses of 'agree' and 'strongly agree.' Agreement with these statements was categorized as either 'strongly disagree,' 'disagree,' 'neither agree nor disagree,' 'agree,' or 'strongly agree'

'HCP referred patient to a class to help learn more about insulin' (31.6\%, mean level of helpfulness 2.26), and 'Patient sought out information about insulin and realized that insulin might not be as bad as first thought' (40.0\%, mean level of helpfulness 2.35). 
Table 3 Participants' reactions when insulin was first recommended, and their insulin-taking behavior

\begin{tabular}{|c|c|}
\hline $\begin{array}{l}\text { Participants' reactions when insulin was first } \\
\text { recommended and their insulin-taking behavior }\end{array}$ & $\begin{array}{l}\text { Frequency } \\
n(\%) \\
(N=99)\end{array}$ \\
\hline \multicolumn{2}{|c|}{$\begin{array}{l}\text { Level of surprise when HCP first said it was time to take insulin, } \\
n(\%)\end{array}$} \\
\hline Not surprised at all & $26(26.3)$ \\
\hline Slightly surprised & $33(33.3)$ \\
\hline Moderately surprised & $28(28.3)$ \\
\hline Very surprised & $12(12.1)$ \\
\hline \multicolumn{2}{|c|}{$\begin{array}{l}\text { Level of being upset when HCP first said it was time to take } \\
\text { insulin, } n(\%)\end{array}$} \\
\hline Not upset at all & $22(22.2)$ \\
\hline Slightly upset & $35(35.4)$ \\
\hline Moderately upset & $29(29.3)$ \\
\hline Very upset & $13(13.1)$ \\
\hline \multicolumn{2}{|c|}{ Willingness to start insulin when first recommended, $n(\%)$} \\
\hline Not willing & $59(59.6 \%)$ \\
\hline \multicolumn{2}{|c|}{$\begin{array}{l}\text { Time between the first recommendation of insulin and the start } \\
\text { of regular insulin use, } n(\%)\end{array}$} \\
\hline I started taking it right away & $80(80.8)$ \\
\hline Less than 1 week & $1(1.0)$ \\
\hline About 1 or 2 weeks & $2(2.0)$ \\
\hline bout 1 month & $4(4.0)$ \\
\hline $2-3$ months & $4(4.0)$ \\
\hline More than 1 year & $8(8.1)$ \\
\hline Taking insulin regularly & $91(91.9)$ \\
\hline $\begin{array}{l}\text { Stopped insulin for a period of } 7 \text { or more days since } \\
\text { first starting insulin, } n(\%)\end{array}$ & $8(8.1)$ \\
\hline $\begin{array}{l}\text { During the first month after I first started taking } \\
\text { insulin }\end{array}$ & $0(0.0)$ \\
\hline 1-3 months after I first started taking insulin & $2(25.0)$ \\
\hline 3-6 months after I first started taking insulin & $3(37.5)$ \\
\hline $\begin{array}{l}\text { More than } 6 \text { months after I first started taking } \\
\text { insulin }\end{array}$ & $3(37.5)$ \\
\hline
\end{tabular}

$H C P$ healthcare provider, $n / N$ number of participants

\section{DISCUSSION}

Possible consequences of delayed insulin use for patients with T2D include serious health complications such as heart disease, stroke, kidney failure, vision loss, and nerve damage [1]. It is therefore imperative that HCPs understand psychological insulin resistance in patients so that adequate measures can be taken to reduce the delay in insulin initiation.

We report a Japanese subpopulation analysis of EMOTION, a multinational survey of current insulin-using adults with T2D. Approximately $40 \%$ of all Japanese participants reported being moderately or very surprised with the recommendation to commence insulin, with approximately $42 \%$ being moderately or very upset. Despite an initial unwillingness to initiate insulin therapy, approximately $80 \%$ of Japanese participants initiated insulin treatment immediately. Notably, the majority of the participants continued to use insulin once they initiated it. This high uptake rate may reflect Japanese participants' trust in their HCPs, a trait associated with increased insulin adherence and reduced diabetes-related stress [22].

Psychosocial factors affecting insulin usage appear to be common among diabetic patients worldwide [13], and educational programs related to insulin initiation differ between Japanese and non-Japanese physicians [23]. Our results indicate that HCP actions are significantly associated with a patient's decision to initiate insulin. We identify a number of HCP actions/events deemed to be the most and least helpful for initiating insulin among reluctant Japanese adults with T2D. Namely, HCP actions that provided practical demonstrations on how to use insulin were the most helpful. Reassurance concerning pain of injection, explanations regarding blood glucose numbers and future health, encouragement to make contact in case of any problems, and occurrence of a positive life event were also rated highly by Japanese participants as being helpful in deciding to start insulin treatment. In addition, some actions occurred less frequently but were considered very useful by Japanese participants. In particular, 'HCP gave an injection while patient was in the office' was not an action performed by many HCPs $(25.3 \%)$, but was rated as helping moderately or a lot by $72.0 \%$ of participants. Similarly, 'Patient talked with someone who was taking insulin and realized it might not be as bad as patient thought' did not occur often $(16.2 \%)$, but when it did occur, $62.5 \%$ of participants found this helped moderately or a lot. 
HCP actions and life events rated as least helpful were those that referred the patient elsewhere, including HCP referral to educational classes and meeting other patients. Additionally, communication style may be important for Japanese patients. For example, 'HCP warned patient that he/she was likely to develop complications if the patient didn't get started with insulin to control his/her diabetes' was only helpful for approximately half of participants (49.2\%). Similarly, 'HCP warned patient that he/she could not be responsible for what might happen if he/she did not start insulin soon' was only helpful to $39.1 \%$ of Japanese participants.

Recent studies have examined reluctance to initiate insulin treatment in various populations. It has been documented that patients who were nonadherent to insulin regimens frequently felt that their HCP did not adequately explain the benefits and risks of insulin treatment [12]. Perceived physician inattention and lack of engagement as well as diabetes-related distress affect insulin adherence and glycemic control [24]. Many factors, including information, adequate counseling, and positive role models, have been found to influence PIR [25]. A multinational study that included Japan examined experiences during and after insulin initiation and discovered that patients who were classified as treatment 'interrupters' or 'discontinuers' were more likely to have concerns and challenges during the initiation process [26]. These patients were also less likely to exhibit improvements in glycemic control compared to patients classified as treatment 'continuers' [26]. In addition, a recent multicenter Japanese study concluded that educating patients about the benefits of insulin therapy may help reluctant insulin users to initiate therapy, and that practical support may assist current insulin users in maintaining therapy [20]. Indeed, our results relating to the helpfulness of HCP actions support these findings and highlight the important role that HCPs play in preventing and/or overcoming PIR amongst patients with T2D.

The Diabetes Attitudes, Wishes and Needs (DAWN2) survey was a global initiative aimed at assessing diabetes care and management among

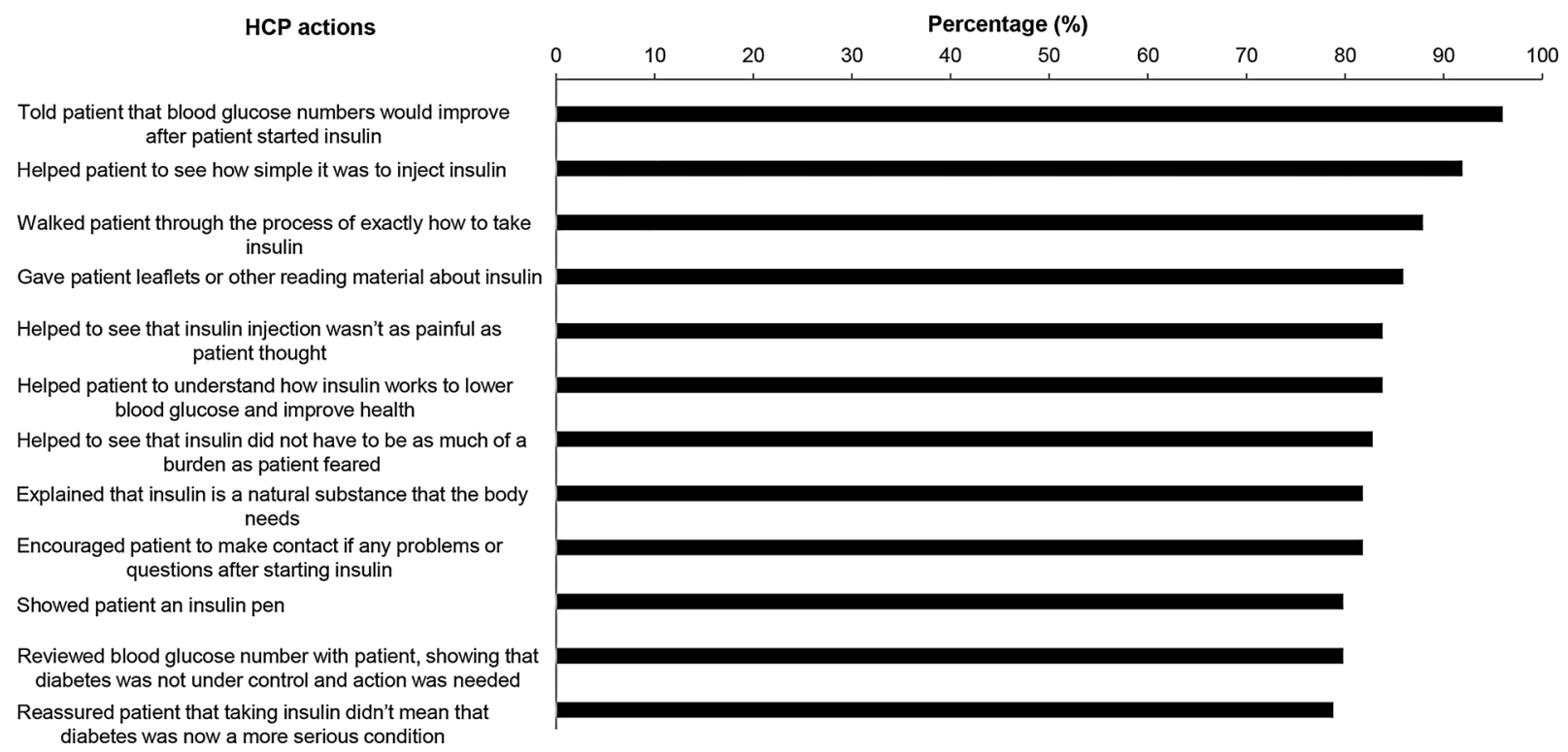

Fig. 1 HCP actions and life events most commonly experienced by Japanese participants with T2D. The 12 most common HCP actions experienced by Japanese participants with T2D are shown. Life events were

considered, but they were not amongst the actions/events most commonly reported by Japanese participants. HCP healthcare provider, T2D type 2 diabetes mellitus 


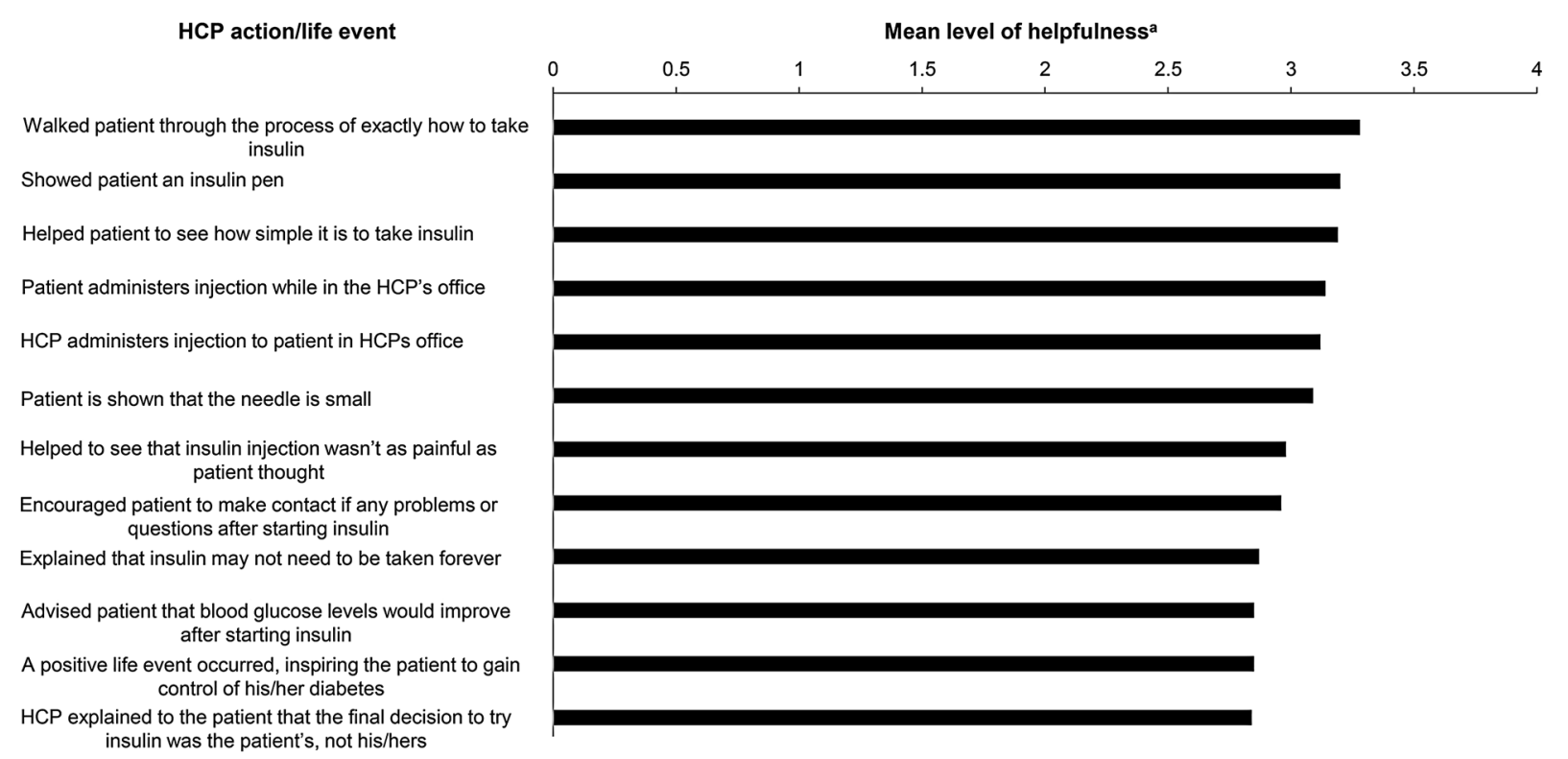

Fig. 2 HCP actions and life events rated as most helpful to Japanese participants with T2D in deciding whether to initiate insulin therapy. The 12 most helpful HCP actions/events experienced by T2D Japanese participants in deciding whether to initiate insulin therapy are shown.

people with diabetes, as well as their HCPs and family members. DAWN2 was conducted in 17 countries, including Japan [23, 27]. In all countries surveyed, DAWN2 revealed that diabetes is a significant physical and psychological burden for many individuals with diabetes [23]. Specifically, DAWN2 reported depression (13.8\%), diabetes-related distress $(44.6 \%)$, poor or very poor quality of life $(12.2 \%)$, a negative impact on relationships (20.5\%) and physical health $(62.2 \%)$, and medication interference in daily life (approximately 40\%) [23]. Many factors may have influenced the findings from DAWN2, such as socioeconomic and cultural factors as well as differences in healthcare systems between countries. Indeed, in our analysis, there are notable differences between Japanese and Caucasian participants from the global EMOTION analysis. For example, differences in the level of full-time employment (51.5\% Japan vs $35.0 \%$ US) and education (participants who completed high school: $42.4 \%$ Japan vs $18.3 \%$ US) were observed. Primary care physicians in Japan were more likely to be involved in assisting patients with initiating insulin
$H C P$ healthcare provider, $T 2 D$ type 2 diabetes mellitus. ${ }^{\text {a}}$ Mean level of helpfulness based on a 4-point scale; $1=$ it didn't help at all, $2=$ it helped a little, $3=$ it helped moderately, $4=$ it helped a lot

compared to the overall EMOTION population (76.8\% Japan vs $37.4 \%$ overall population), for whom this task was also likely to be performed by endocrinologists (12.6\%) and diabetes nurse specialists (14.0\%). Hence, country-specific analyses are warranted.

Diabetes-related distress, known as the emotional burden of and reaction to managing a chronic illness, is associated with a higher rate of diabetes-related complications [23, 28, 29]. A positive educational experience and support from HCPs at the time of insulin recommendation may be pivotal to maintaining adequate glycemic control and the long-term health of patients with T2D. In fact, a large retrospective cohort study found that a substantial proportion of people continue with poor glycemic control for several years before initiating intensification with oral antidiabetic medications and insulin [30]. Diabetes health-coaching consisting of goal-setting, education, and individualized and follow-up care have also been effective strategies to improve glycemic control, as demonstrated in a recent meta-analysis which concluded that diabetes health-coaching 
resulted in a statistically significant reduction in HbA1c levels $(0.32 \%)$ [31]. Physician empathy is also noted as contributing to improved HbA1c outcomes [32], and practical strategies that HCPs can utilize to assist their patients to overcome emotional barriers may be warranted (e.g., the use of mnemonics) [33]. Practical diabetes health-coaching, along with routine diabetes care, supports our findings amongst Japanese participants, and may be a valuable tool to help motivate T2D patients to initiate and maintain insulin treatment.

This study provides valuable data regarding the experiences of Japanese participants diagnosed with T2D. We identify HCP actions and life events that were helpful in initiating insulin treatment. Our results agree with recently described results of a stepwise approach to diabetes and insulin education [17], which encompassed problem-solving, patient education, and physician enquiries about patient feelings and fears.

The strength in our analysis lies in the description of helpful (and less helpful) HCP actions rated by Japanese participants with T2D; data which are, at present, limited in this population. Limitations relating to the study design, however, should be noted. Firstly, the majority of survey respondents were recruited from online panels of individuals who had volunteered to participate in studies. These participants, therefore, may be more involved in their own diabetes management in comparison with typical patients. Perceptions regarding helpful PIR actions in these volunteers may not be indicative of the perceptions of the broader population. Secondly, the majority of Japanese survey participants were male and responses may vary with increased female participation. Thirdly, survey responses may be due to recall bias, with participants having initiated insulin between 2015 and 2017. Finally, the retrospective nature of the study should raise some caution: self-reported data may not be accurate and could potentially be subject to bias.

Further studies amongst this patient population are warranted and may further assist HCPs in providing optimal care for Japanese patients with T2D. Other factors that may affect PIR in Japanese patients, such as age, socioeconomic status, and mental health, also warrant further investigation.

\section{CONCLUSION}

Our findings suggest that a tailored approach in which HCPs explain the insulin injection process from patients' perspectives is beneficial for reluctant Japanese insulin users. This study provides important information on actions that can be used by HCPs to assist Japanese adults with T2D who are reluctant insulin users in overcoming PIR. More generally, the findings from this survey of Japanese adults with T2D, who were initially reluctant to begin insulin treatment, suggest potentially useful clinical interventions for helping patients overcome PIR, a critical step towards designing comprehensive clinical recommendations for HCPs.

\section{ACKNOWLEDGEMENTS}

We thank the participants and the study personnel who participated in this study. Project management support was provided by Aki Yoshikawa from Eli Lilly Japan K.K.

Funding. This study was sponsored by Eli Lilly and Company (Indianapolis, IN, USA) and Boehringer Ingelheim (Ingelheim, Germany). Eli Lilly Japan K.K. (Kobe, Japan) and Nippon Boehringer Ingelheim Co., Ltd. (Tokyo, Japan) funded the Rapid Service Fee. All authors had full access to all of the data in this study and take complete responsibility for the integrity of the data and accuracy of the data analysis.

Medical Writing and Editorial Assistance. Medical writing support was provided by Lisa Cossens and editorial support was provided by Angela Lorio, both of Syneos Health. Medical writing and editorial assistance was funded by Eli Lilly and Company.

Authorship. All named authors meet the International Committee of Medical Journal Editors (ICMJE) criteria for authorship for this article, take responsibility for the integrity of 
the work as a whole, and have given their approval for this version to be published.

Disclosures. Kentaro Okazaki reports an advisory role with Eli Lilly and Company, Japan K.K. Tomotaka Shingaki is an employee and stockholder at Eli Lilly Japan K.K. Zhihong Cai is an employee and stockholder at Eli Lilly Japan K.K. Magaly Perez-Nieves is an employee and stockholder at Eli Lilly and Company. Lawrence Fisher serves as an advisor for Eli Lilly and Company and has received travel grants from Eli Lilly and Company.

Compliance with Ethics Guidelines. The study protocol was approved by the Western Institutional Review Board (IRB), Puyallup, Washington, USA, as well as the Nagoya University IRB, Nagoya, Japan. All procedures performed in this study involving human participants were in accordance with the ethical standards of the Western IRB, Puyallup, Washington, USA, and Nagoya University IRB, Nagoya, Japan, and with the 1964 Declaration of Helsinki and its later amendments or comparable ethical standards. Informed consent was obtained from all individual participants included in the study. Participants were compensated for their time.

Data Availability. The datasets of the current study are not publicly available. Eli Lilly and company provides access to individual patient data from studies on approved medicines and indications as defined by the sponsorspecific information at http://clinicalstudydata request.com. Researchers require an approved research proposal submitted through http:// clinicalstudydatarequest.com. Access to data will be provided in a secure data sharing environment after signing a data sharing agreement.

Open Access. This article is distributed under the terms of the Creative Commons Attribution-NonCommercial 4.0 International License (http://creativecommons.org/licenses/ by-nc/4.0/), which permits any noncommercial use, distribution, and reproduction in any medium, provided you give appropriate credit to the original author(s) and the source, provide a link to the Creative Commons license, and indicate if changes were made.

\section{REFERENCES}

1. World Health Organization. Global report on diabetes. 2016. http://www.who.int/diabetes/globalreport/en/. Accessed 18 June 2018.

2. Goto A, Noda $M$, Inoue $M$, Goto $M$, Charvat $H$. Increasing number of people with diabetes in Japan: is this trend real? Intern Med. 2016;55(14):1827-30.

3. Davies A, D'Alessio D, Fradkin J, et al. Management of hyperglycaemia in type 2 diabetes, 2018. A consensus report by the American Diabetes Association (ADA) and the European Association for the Study of Diabetes (EASD). Diabetologia. 2018;61:2461-98.

4. Haneda $M$, Noda $M$, Origasa $H$, et al. Japanese clinical practice guideline for diabetes 2016. J Diabetes Investig. 2018;9:657-97. https://doi.org/10. 1111/jdi.12810.

5. Polonsky WH, Fisher L, Guzman S, Villa-Caballero L, Edelman SV. Psychological insulin resistance in patients with type 2 diabetes: the scope of the problem. Diabetes Care. 2005;28:2543-5.

6. Hosomura N, Malmasi S, Timerman D, et al. Decline of insulin therapy and delays in insulin initiation in people with uncontrolled diabetes mellitus. Diabet Med. 2017;34:1599-602.

7. Asakura T, Suzuki S, Aranishi T, Cai Z. Comparative usability study of the dulaglutide single-use pen versus the insulin degludec FlexTouch (R) among self-injection-naive patients with type 2 diabetes mellitus in Japan. Curr Med Res Opin. 2018;34:1117-24.

8. Gelhorn HL, Bacci ED, Poon JL, Boye KS, Suzuki S, Babineaux SM. Evaluating preferences for profiles of glucagon-like peptide-1 receptor agonists among injection-naive type 2 diabetes patients in Japan. Patient Prefer Adher. 2016;10:1337-48.

9. Polonsky WH, Hajos TR, Dain MP, Snoek FJ. Are patients with type 2 diabetes reluctant to start insulin therapy? An examination of the scope and underpinnings of psychological insulin resistance in a large, international population. Curr Med Res Opin. 2011;27:1169-74.

10. Brod M, Kongsø JH, Lessard S, Christensen TL. Psychological insulin resistance: patient beliefs and implications for diabetes management. Qual Life Res. 2009;18:23-32. 
11. Larkin ME, Capasso VA, Chen CL, et al. Measuring psychological insulin resistance: barriers to insulin use. Diabetes Educ. 2008;34:511-7.

12. Karter AJ, Subramanian U, Saha C, et al. Barriers to insulin initiation: the translating research into action for diabetes insulin starts project. Diabetes Care. 2010;33:733-5.

13. Peyrot M, Rubin RR, Lauritzen T, et al. Resistance to insulin therapy among patients and providers: results of the cross-national Diabetes Attitudes, Wishes, and Needs (DAWN) study. Diabetes Care. 2005;28:2673-9.

14. Brod M, Alolga SL, Meneghini L. Barriers to initiating insulin in type 2 diabetes patients: development of a new patient education tool to address myths, misconceptions and clinical realities. Patient. 2014;7:437-50.

15. Krall J, Gabbay R, Zickmund S, Hamm ME, Williams KR, Siminerio L. Current perspectives on psychological insulin resistance: primary care provider and patient views. Diabetes Technol Ther. 2015;17:268-74.

16. Polonsky WH, Jackson RA. What's so tough about taking insulin? Addressing the problem of psychological insulin resistance in type 2 diabetes. Clin Diabetes. 2004;22(3):147-50.

17. Polonsky WH, Arsenault J, Fisher L, et al. Initiating insulin: How to help people with type 2 diabetes start and continue insulin successfully. Int J Clin Pract. 2017;71(8). https://doi.org/10.0000/ijcp. 12973.

18. Chuman N, Doi Y. Transition of psychological and behavioral reactions to insulin treatment in patients with type 2 diabetes. J Jpn Soc Nurs Res. 2011;34(5):59-69.

19. Yoshioka N, Ishii $\mathrm{H}$, Tajima N, Iwamoto $\mathrm{Y}$, DAWN Japan Group. Differences in physician and patient perceptions about insulin therapy for management of type 2 diabetes: the DAWN Japan study. Curr Med Res Opin. 2014;30:177-83.

20. Odawara M, Ishii H, Tajima N, Iwamoto Y. Impact of patient attitudes and beliefs to insulin therapy upon initiation, and their attitudinal changes after initiation: the DAWN Japan study. Curr Med Res Opin. 2016;32:681-6.

21. Polonsky WH, Fisher L, Hessler D, et al. Identifying solutions to psychological insulin resistance: an international study. J Diabetes Complicat. 2019;33:307-14. jdiacomp.2019.01.001.
22. Halepian L, Saleh MB, Hallit S, et al. Adherence to insulin, emotional distress, and trust in physician among patients with diabetes: a cross-sectional study. Diabetes Ther. 2018;9:731-826.

23. Nicolucci A, Kovacs Burns K, Holt RI, et al. Diabetes attitudes, wishes and needs second study (DAWN2): cross-national benchmarking of diabetes-related psychosocial outcomes for people with diabetes. Diabet Med. 2013;30:767-77.

24. Linetzky B, Jiang D, Funnell MM, et al. Exploring the role of the patient-physician relationship on insulin adherence and clinical outcomes in type 2 diabetes: insights from the MOSAIc study. J Diabetes. 2017;9:596-605.

25. Lee KP. Psychosocial factors associated with psychological insulin resistance in primary care patients in Hong Kong. J Clin Transl Endocrinol. 2015;2:157-62.

26. Perez-Nieves M, Ivanova JI, Hadjiyianni I, et al. Basal insulin initiation use and experience among people with type 2 diabetes mellitus with different patterns of persistence: results from a multi-national survey. Curr Med Res Opin. 2017;33:1833-42.

27. Peyrot M, Burns KK, Davies M, et al. Diabetes Attitudes, Wishes and Needs 2 (DAWN2): a multinational, multi-stakeholder study of psychosocial issues in diabetes and person-centred diabetes care. Diabetes Res Clin Pract. 2013;99:174-84.

28. Fisher L, Hessler D, Glasgow RE, et al. REDEEM: a pragmatic trial to reduce diabetes distress. Diabetes Care. 2013;26:2551-8.

29. Ascher-Svanum H, Zagar A, Jiang D, et al. Associations between glycemic control, depressed mood, clinical depression, and diabetes distress before and after insulin initiation: an exploratory, post hoc analysis. Diabetes Ther. 2015;6:303-16.

30. Khunti K, Wolden ML, Thorsted BL, et al. Clinical inertia in people with type 2 diabetes: a retrospective cohort study of more than 80,000 people. Diabetes Care. 2013;36:3411-7.

31. Sherifali D, Viscardi V, Bai JW, et al. Evaluating the effect of a diabetes health coach in individuals with type 2 diabetes. Can J Diabetes. 2016;40:84-94.

32. Hojat M, Louis DZ, Markham FW, et al. Physicians' empathy and clinical outcomes for diabetic patients. Acad Med. 2011;86:389-464.

33. Kalra S, Verma K. Handling insulin-related emotions. Diabetes Ther. 2018;9:1415-9. 